\title{
Report
}

\section{Ultraconserved Elements in the Human Genome}

\author{
Gill Bejerano, ${ }^{1 *}$ Michael Pheasant, ${ }^{3}$ Igor Makunin, ${ }^{3}$ Stuart Stephen, ${ }^{3}$ W. James Kent, ${ }^{1}$ John S. Mattick, ${ }^{3}$ \\ David Haussler ${ }^{2 *}$
}

${ }^{1}$ Department of Biomolecular Engineering, ${ }^{2}$ Howard Hughes Medical Institute, University of California Santa Cruz, Santa Cruz, CA 95064, USA. ${ }^{3}$ ARC Special Research Centre for Functional and Applied Genomics, Institute for Molecular Bioscience, University of Queensland, Brisbane, QLD 4072, Australia.

*To whom correspondence should be addressed. E-mail: jill@ soe.ucsc.edu (G.B); haussler@ soe.ucsc.edu (D.H.).

There are 481 segments longer than 200 bp that are absolutely conserved $(100 \%$ identity with no insertions or deletions) between orthologous regions of the human, rat and mouse genomes. Nearly all of these segments are also conserved in the chicken and dog genomes, with an average of $95 \%$ and $99 \%$ identity, respectively. Many are also significantly conserved in fish. These ultraconserved elements of the human genome are most often located either overlapping exons in genes involved in RNA processing or in introns or nearby genes involved in regulation of transcription and development. Along with more than 5,000 sequences of over 100bp that are absolutely conserved among the three sequenced mammals, these represent a class of genetic elements whose functions and evolutionary origins are yet to be determined, but which are more highly conserved between these species than proteins, and appear to be essential for the ontogeny of mammals and other vertebrates.

Although only about $1.2 \%$ of the human genome appears to code for protein (1-3), it has been estimated that as much as $5 \%$ is more conserved than expected from neutral evolution since the split with rodents, and hence may be under negative or "purifying" selection (4-6). Several studies have found specific non-coding segments in the human genome that appear to be under selection, using a threshold for conservation of $70 \%$ or $80 \%$ identity with mouse over more than 100bp (7-13). A study of these elements on human chromosome 21 found that those that were very highly conserved in multiple species contained significant numbers of non-coding elements (13). Similar results were found comparing the human, mouse and rat $(14,15)$ in a study of the $1.8 \mathrm{Mb}$ CFTR region $(16,17)$, and in a functional study of the SIM2 locus in a number of mammalian species (18). We determined the longest segments of the human genome that are maximally conserved with orthologous segments in rodents: those showing $100 \%$ identity and with no insertions or deletions in their alignment with mouse and rat. Exclusive of ribosomal RNA regions, there are 481 such segments longer than 200bp that we call ultraconserved elements (table S1). They are widely distributed in the genome (on all chromosomes except chromosomes 21 and $\mathrm{Y}$ ), and are often found in clusters (Fig. 1). The probability is less than $10^{-22}$ of finding even one such element in 2.9 billion bases under a simple model of neutral evolution with independent substitutions at each site, using the slowest neutral substitution rate that is observed for any $1 \mathrm{Mb}$ region of the genome (supporting text, section S1). Nearly all of these elements also exhibited extremely high levels of conservation with orthologous regions in the chicken genome (467/481= $97 \%$ of the elements aligning at an average of $95.7 \%$ identity, 29 at $100 \%$ identity), and about two-thirds of them with the fugu genome as well $(324 / 481=67.3 \%$ of the elements aligning at an average of $76.8 \%$ identity), despite the fact that only about $4 \%$ of the human genome can be reliably aligned to the chicken genome (at an average of $62.9 \%$ identity where an alignment is found) and less than $1.8 \%$ of the human genome aligns to fugu (at an average of $60 \%$ identity). In addition, nearly all exhibited extremely high levels of conservation with the dog genome, estimated using reads from the NCBI trace archive $(477 / 481=99.2 \%$ of the elements aligning at an average of $99.2 \%$ identity).Thus it appears that nearly all of these ultraconserved elements may have been under extreme negative selection in many species for more than 300 million years, and some of them for at least 400 million years.

As expected, the ultraconserved elements exhibit almost no natural variation in the human population. Only 6 out of 106,767 bases examined in the ultraconserved elements (excluding the first and last 20 bases in each element) are at validated SNPs in dbSNP (table S2a). For this much DNA we would have expected 119 validated sites, so validated SNPs are under-represented by 20 -fold $\left(P<10^{-42}\right)$. The 48 unvalidated SNPs we found revealed many likely errors in the unvalidated portion of the dbSNP database (table S2b). These same 106,767 bases exhibit very few differences with the 
chimp genome as well, showing only 38 single base changes where the chimp base has Phred quality score at least 45 , whereas the expected number would be 716 (roughly 19-fold reduction, $P<10^{-200}$, supporting text, section $\mathrm{S} 2$ ). This low level of variation within the human population and in comparison with chimp suggests that these elements are currently changing at a rate that is roughly 20 times slower than the average for the genome. Only $4.3 \%$ of the bases are different in chicken, which is also consistent with a roughly 20 -fold reduction over neutral substitution rates (supporting text, section $\mathrm{S} 2$ ).

Of the 481 ultraconserved elements, 111 overlap the mRNA of a known human protein coding gene (including the UTR regions), 256 show no evidence of transcription from any matching EST or mRNA from any species, and for the remaining 114 the evidence for transcription is inconclusive. We call these partly exonic (or "exonic" for short), nonexonic, and possibly exonic ultraconserved elements, respectively. A hundred non-exonic elements are located in introns of known genes and the rest are intergenic. The nonexonic elements, both intronic and intergenic, tend to congregate in clusters near transcription factors and developmental genes (further analysis below), whereas the exonic and possibly exonic elements are more randomly distributed along the chromosomes (Fig. 1).

There are 93 known genes that overlap with exonic ultraconserved elements; we call these type I genes. The 255 genes that are nearby the non-exonic elements we call type II (methods in supporting text, section S3). We looked for categories of biological process and molecular function defined in the Gene Ontology (GO) database (19) that are significantly enriched in type I and II genes, and also searched InterPro (20) for enrichment in particular structural domains (Fig. 2). The type I genes show significant functional enrichment for RNA binding and regulation of splicing $\left(P<10^{-18}\right.$ and $10^{-9}$, respectively, against all GO annotated human genes) and are uniquely abundant in the RNA recognition motif, RRM, $\left(P<10^{-17}\right.$, against all InterPro annotated human genes). In contrast, the type II genes are devoid of enrichment for RNA binding, splicing or the RRM ( $P=0.39,0.44,0.77$, respectively). However, type II genes are strongly enriched for regulation of transcription and DNA binding $\left(P<10^{-19}\right.$ and $10^{-14}$, respectively), as well as DNA binding motifs, in particular the Homeobox $\left(P<10^{-14}\right)$. These three attributes are enriched in type I genes as well, but 16, 8 and 9 orders of magnitude less significantly, respectively. This suggests that exonic ultraconserved elements may be specifically associated with RNA processing and non-exonic with regulation of transcription at the DNA level.

Non-exonic ultraconserved elements are often found in "gene deserts" that extend more than a megabase. In particular, of the non-exonic elements, there are 140 that are more than $10 \mathrm{~Kb}$ away from any known gene, and 88 that are more than $100 \mathrm{~Kb}$ away. The set of 156 annotated genes that flank intergenic ultraconserved elements is significantly enriched for developmental genes $\left(P<10^{-6}\right)$, and in particular genes involved in early developmental tasks $\left(P=2.7 \times 10^{-5}\right)$, suggesting many of the associated ultraconserved elements may be distal enhancers of these early developmental genes. Indeed, one of these elements (uc.351 in table S1) is contained in an enhancer situated about $225 \mathrm{~Kb}$ upstream of DACH (homolog of Drosophila dachshund gene, known to be involved in the development of brain, limbs and sensory organs), which has been shown to reproducibly drive expression in the retina when cloned upstream of a mouse heat shock protein 69 minimal promoter coupled to betagalactosidase and injected into a mouse oocyte (21). Nonexonic ultraconserved elements that lie in introns are also often associated with developmental genes. These include the neuroretina-specific enhancer in the $4^{\text {th }}$ intron of PAX6 (uc.328), investigated in quail but shown to also be functionally conserved in mouse (22).

Type I genes (harboring exonic elements) include many genes encoding well-known RNA binding proteins, such as HNRPK, HNRPH1, HNRPU, HNRPDL, HNRPM, SFRS1, SFRS3, SFRS6, SFRS7, SFRS10, SFRS11, TRA2A, PCBP2 and PTBP2. All of the above are among the 59 type I genes annotated by GO which exhibit clear mRNA/EST evidence of alternative splicing overlapping the ultraconserved element (out of 68 elements in all, from a total of 111 exonic elements, table S3). Many of the above, including the six members of the SFRS family, contain the RNA recognition motif. The ultraconserved elements associated with alternative splicing events often contain small coding exons that are skipped in the mRNA in some tissues, but the elements extend well into the flanking intronic regions on one or both sides of the exon. Such is the case for one explicitly studied ultraconserved element (uc.33) in PTBP2, a polypyrimidine tract binding protein (23). PTBP2 contains a 312 bp ultraconserved segment that is mostly intronic, but includes a small (34bp) exon that is included in the mRNA only in brain tissue. The 203 bases at the 3' end of the element, including the $34 \mathrm{bp}$ exon, are $100 \%$ conserved in chicken as well.

The PTBP2 element may form an RNA structure in the pre-mRNA that participates in the regulation of splicing through interactions with the spliceosome (23). We used RNAfold (24) to further assess the potential of this and other ultraconserved elements to form an RNA secondary structure, comparing the energy of the best folded structure for both the positive and negative strand element to that of 10,000 random permutations of the same sequence (table S4). No statistically significant structure was found for the PTBP2 element, but the energy of the fold for the $573 \mathrm{bp}$ ultraconserved self- 
regulated alt-spliced UTR element (uc.189) in arginine/serine-rich splicing factor SFRS3 (25) was lower than that of all but one of the 10,000 randomized versions of this sequence, indicating that it may form an important RNA secondary structure (fig. S2).

In addition to alternative splicing, the exonic ultraconserved elements also include the consecutive, mutually exclusive "flop" and "flip" exons (uc.478/9) from the glutamate receptor GRIA3 (26), which exhibits RNA editing as well as alternate splicing (27). The "flop" ultraconserved element extends into the $\sim 600 \mathrm{bp}$ intron 13 of the gene. At the other end (adjacent to the previous exon), intron 13 contains a much shorter highly conserved RNA hairpin structure that guides the essential and highly regulated editing of adenosine to inosine (27). While the element containing the "flop" exon does not have detectable RNA secondary structure preferences, the minimal energy of the secondary structure of the element containing the "flip" is less than that of 34 out of 10,000 permuted versions, indicating possible structure.

Although the minimal region of $100 \%$ conservation between human, mouse and rat that was required to be included in the ultraconserved set was $200 \mathrm{bp}$, many elements were considerably longer. The longest elements (779bp, $770 \mathrm{bp}$, and 731bp) all lie in the last three introns in the 3 ' portion of POLA, the DNA polymerase alpha catalytic subunit (EC 2.7.7.7) on chromosome X, along with other shorter ultraconserved elements (Fig. 1). A similar-sized conserved region, $711 \mathrm{bp}$ formed by concatenation of uc.468 and uc.469 (separated by a single base), lies in the $\sim 7 \mathrm{~Kb}$ intergenic region between the 3' end of POLA and its downstream neighbor, the ARX homeobox gene. ARX is involved in CNS development and is associated with a host of $\mathrm{X}$-linked Mendelian diseases, including epilepsy, mental retardation, autism and cerebral malformations (28). Because this group of elements lies at the 3' end of the $303 \mathrm{~kb}$ POLA gene, nearer to the 3' end of ARX than to the rest of POLA (Fig. 1), it is possible that their function is not related to POLA, but that they instead form a cluster of enhancers of ARX. The longest of these ultraconserved elements, $779 \mathrm{bp}$, is actually adjacent to a $275 \mathrm{bp}$ element, which together form a $1046 \mathrm{bp}$ region with only one change in rodents. As a calibration, note that these POLA/ARX elements are considerably longer than the ultraconserved portions of the human, mouse and rat ribosomal RNA genes, which harbor six ultraconserved segments, three each in the $18 \mathrm{~S}$ and $28 \mathrm{~S}$, the longest of which is 563bp (table S1).

In sharp contrast to rRNA and most human coding regions, there were only 24/481 cases (5\%) where an ortholog of an ultraconserved element could be traced back by sequence similarity search as far as Ciona intestinalis, Drosoplila melanogaster, or Caenorhabditis elegans (table S7). All of these were among the 68 elements (14\%) that overlapped coding exons from known genes. In 17 of these 24 "ancient" cases there is clear mRNA or EST evidence that the coding region overlapped by the element is alternatively spliced in human. These include alternatively spliced exons of genes EIF2C1, BCL11A, EVI1, ZFR, CLK4, HNRPH1, and DDX5, as well as GRIA3. In none of the other cases could we find evidence that any element that was intronic in human was coding in another species, although in some cases there was EST evidence for a retained intron that presumably has a function other than protein-coding. Moreover, indels of noncoding ultraconserved elements relative to their alignments with chicken and other species are often not in multiples of three, giving further evidence that these sequences are noncoding (fig. S1, A and B,b).

The ultraconserved elements we found in introns seem to have been at one time rather fast-evolving compared to the known coding exons in their genes. We tried to map selected introns containing ultraconserved intronic elements to more distant species using protein/translated DNA matches to their enclosing exons. Often only a "core" conserved region was recognizable in fish and this had very different flanking DNA, suggesting additional parts of the ultraconserved region were innovations after the common ancestor with fish, as observed in the analysis of uc.108 near HOXD (29). In cases where we could trace beyond vertebrates, we always found that the orthologous intron in the more distant species was either very small with apparently unrelated sequence, or was nonexistent. For example, tracing the intron that contains the first (most 5') ultraconserved element in POLA (uc.460), we find that while it is an approximately $50 \mathrm{~Kb}$ intron in human, its ortholog in Fugu rubripes is only $\sim 7500$ bp (still large relative to most fugu introns), only about 335bp in Ciona intestinalis, and does not exist (the flanking exons abut) in $D$. melanogaster and C. elegans. The human element is not recognizably similar to anything in the orthologous intron of Ciona. Yet like the other POLA ultraconserved elements discussed above, this element is more than $99 \%$ identical between human and chicken. Similar results were found for the three longest POLA intronic elements. Another similar case was a cluster of seven ultraconserved elements (uc.2739) with sizes from $237 \mathrm{bp}$ to $432 \mathrm{bp}$ all contained in an $\sim 165 \mathrm{~kb}$ intron of $\mathrm{PBX} 3$, pre-B-cell leukemia transcription factor 3, a member of the TALE/PBX homeobox family. This was one of the largest introns we found, and contained one of the largest collections of ultraconserved elements in a single intron. The orthologous intron in Fugu rubripes is $\sim 38 \mathrm{~Kb}$, in Ciona intestinalis it appears to be $\sim 1 \mathrm{~Kb}$, in Drosophila 200bp (ortholog exd), and the flanking exons abut in $C$. elegans. Despite the inability to trace most of the vertebrate ultraconserved elements to distant species, the possibility that processes similar to those that produced ultraconserved 
elements in vertebrates also exist in other classes of species remains open. In one tantalizing example, it has been observed that the mating type gene MATa2 in yeast shows $100 \%$ conservation over $357 \mathrm{bp}$ in four yeast species (30). The mechanism of this conservation is not known.

We found only 12 paralogous sets, each consisting of 2-3 elements, among all 481 ultraconserved elements (table S5). Each paralogous set is consistent with the paralogy relationship between the enclosing or nearby "host" genes. All paralogs (except, currently, uc.344 overlapping HOXC5) have highly conserved matches in chicken, providing more opportunities for evolutionary analysis of these duplication events that predate divergence from birds. In each of the clusters we found significant divergence between the paralogs, which must have occurred in the early part of their evolution (fig. S3), as each individual instance in a paralogous set has changed very little in the last 300 million years in birds and mammals. This, combined with the above analysis, suggests that the bulk of the ultraconserved elements represent chordate innovations that evolved fairly rapidly at first but then slowed down considerably, becoming effectively "frozen" in birds and mammals.

A more extensive analysis of paralogs, based on a recent global clustering of highly conserved non-coding human DNA (31), reveals several further highly conserved intronic and intergenic elements in functionally equivalent positions relative to paralogous genes. These were not classified as ultraconserved by our stringent criteria. Indeed, if we merge alignment blocks of 200 bases, each with at least $99 \%$ identical columns, we obtain 1,974 "highly conserved" elements, of length up to $1,087 \mathrm{bp}$ in human. Four of the five longest elements are the aforementioned POLA/ARX elements, along with a 906bp element (encompassing uc.326/7) in an intron of ELP4, adjacent to PAX6. If instead we demand at least a 100bp exact match between human and rodents, we get more than 5,000 highly conserved elements. Tens of thousands more are found at lower cutoffs - for example there is a $57 \mathrm{bp}$ exactly conserved sequence overlapping an alternatively spliced exon of the WT1 gene which is invariant in mammals and in chicken, and is largely conserved in fishes (fig. S1). The percentage of the conserved elements that overlap with a known coding region steadily rises from $14 \%$ to $34.7 \%$ as the length criteria defining these elements is reduced from 200bp to 50bp (table S6).

If experiments with less conserved elements in recent studies $(13,18)$ are any indication, many of these shorter elements are also functional. Compared to the ultraconserved elements, a greater percentage of these shorter conserved elements are significantly different in birds, while highly conserved in mammals. This suggests that the process of evolution of new elements followed by near "freezing" of their DNA sequences is probably still ongoing in vertebrates. Lineage-specific specializations of these elements may reflect regulatory changes that are important to the ontogeny and physiology of the clade.

The patterns of conservation exhibited in the ultraconserved elements must result from the onset during chordate evolution of either a highly elevated negative selection rate in these regions (about 20 times smaller chance of mutations becoming fixed in the population), a highly reduced mutation rate (about 20 times fewer mutations), or some combination of these effects. The possibility of strong negative selection is intriguing because selection to maintain protein coding, protein-nucleic acid interactions, or RNARNA interactions does not result in near total conservation over long stretches of bases unless multiple functions are overlaid on the same DNA, e.g. in regions of coding exons that also bind splicing factors, or in regions of ribosomal RNA that must form RNA structures as well as bind proteins. If the exonic ultraconserved elements form pre-mRNA structures that are under selection to preserve interaction with the spliceosome or editing machinery $(23,27)$, then these interactions must be extremely constraining over hundreds of bases of DNA, much like those of the anciently derived ribosomal RNAs, making them potentially quite novel objects for molecular study. The same holds true if the conservation in the non-exonic elements is associated with selection for molecular interactions involved in the regulation of transcription, which could be in cis over long genomic distances, or in trans, perhaps also involving RNA (29, 32, 33).

On the other hand, if reduced mutation rates is the explanation, then the existence of regions of a few hundred bases with 20 -fold reduced mutation rates would itself be quite novel. Although neutral mutation rates may vary depending on chromosomal location on a megabase scale (34-36), there is to our knowledge no evidence or precedent for the existence of short "hypo-mutable" or "hyper-repaired" neutral regions. Finally, the answer can also be a combination of negative selection and better repair in these regions owing to some vital role that these elements play, such as selfregulating networks of RNA processing control in the case of exonic elements and self regulatory networks of transcriptional control for non-exonic elements. In any case, the questions remain - what kind of elements associated with these processes would have arrived relatively early in chordate evolution and then become practically frozen in birds and mammals? And what mechanisms would underlie this, allowing them to resist virtually all further change?

Note added in proof: We recently became aware of related observations made by Boffelli et al. (37). 


\section{References and Notes}

1. E. S. Lander et al., Nature 409, 860 (2001).

2. J. C. Venter et al., Science 291, 1304 (2001).

3. Human Genome Sequencing Consortium, in preparation.

4. R. H. Waterston et al., Nature 420, 520 (2002).

5. K. M. Roskin, M. Diekhans, D. Haussler, in Proceedings of the 7th Annual International Conference on Research in Computational Molecular Biology (2003).

6. F. Chiaromonte et al., Cold Spring Harbor Symp. Quant. Biol. (2003).

7. R. C. Hardison, Trends Genet. 16, 369 (2000).

8. G. G. Loots et al., Science 288, 136 (2000).

9. L. A. Pennacchio, E. M. Rubin, Nature Rev. Genet. 2, 100 (2001).

10. K. A. Frazer et al., Genome Res. 11, 1651 (2001).

11. U. DeSilva et al., Genome Res. 12, 3 (2002).

12. E. T. Dermitzakis et al., Nature 420, 578 (2002).

13. E. T. Dermitzakis et al., Science 302, 1033 (2003).

14. Rat Genome Sequencing Consortium, Nature 428, 493 (2004).

15. G. M. Cooper et al., Genome Res. 14, 539 (2004).

16. J. W. Thomas et al., Nature 424, 788 (2003).

17. E. H. Margulies, M. Blanchette, D. Haussler, E. D. Green, Genome Res. 13, 2507 (2003).

18. K. A. Frazer et al., Genome Res. (2004).

19. M. Ashburner et al., Nature Genet. 25, 25 (2000).

20. N. J. Mulder et al., Nucleic Acids Res. 31, 315 (2003).

21. M. A. Nobrega, I. Ovcharenko, V. Afzal, E. M. Rubin, Science 302, 413 (2003).

22. S. Plaza, C. Dozier, M. C. Langlois, S. Saule, Mol. Cell Biol. 15, 892 (1995).

23. L. Rahman, V. Bliskovski, F. J. Kaye, M. Zajac-Kaye, Genomics 83, 76 (2004).

24. I. L. Hofacker, Nucleic Acids Res. 31, 3429 (2003).

25. H. Jumaa, P. J. Nielsen, EMBO J. 16, 5077 (1997).

26. B. Sommer et al., Science 249, 1580 (1990).

27. P. J. Aruscavage, B. L. Bass, RNA 6, 257 (2000).

28. E. H. Sherr, Curr. Opin. Pediatr. 15, 567 (2003).

29. C. Sabarinadh, S. Subramanian, R. Mishra, Genome Biol. 4 (2003).

30. M. Kellis, N. Patterson, M. Endrizzi, B. Birren, E. S. Lander, Nature 423, 241 (2003).

31. G. Bejerano, D. Haussler, M. Blanchette, Proc. Intelligent Systems in Molecular Biology and Bioinformatics, in press.

32. J. S. Mattick, M. J. Gagen, Mol. Biol. Evol. 18, 1611 (2001).

33. E. T. Dermitzakis et al., Genome Res. (2004).

34. K. H. Wolfe, P. M. Sharp, W. H. Li, Nature 337, 283 (1989).

35. R. C. Hardison et al., Genome Res. 13, 13 (2003).

36. J. H. Chuang, H. Li, PLoS Biol. 2, E29 (2004).
37. D. Boffelli, M. Nobrega, E. M. Rubin, Nature Rev. Genet., in press.

38. F. Spitz, F. Gonzalez, D. Duboule, Cell 113, 405 (2003).

39. We thank the Genome Sequencing Consortia for the human, mouse, rat and other genome sequences we used in this analysis. We thank W. Miller, M. Diekhans, A. Hinrichs, K. Rosenbloom, D. Thomas and the members of the UCSC browser team for providing the genome alignments and other tracks of genome annotation available on the UCSC genome browser. We also thank M. Blanchette, S. Salama, T. Lowe, M. Ares, K. Pollard, and B. Cohen for helpful discussions, A. Siepel for the neutral substitution rate analysis involving chicken and chimp, $\mathrm{K}$. Roskin for the calculation of the percent identity in ancestral repeat sites for $1 \mathrm{Mb}$ windows, and $\mathrm{S}$. Walton for help in preparing the manuscript. G.B., W.J.K., and D.H. were supported by NHGRI grant 1P41HG02371, NCI contract 22XS013A, and D.H. additionally by the Howard Hughes Medical Institute. S.S., M.P., I.M., and J.S.M. were supported by the Australian Research Council and the Queensland State Government.

\section{Supporting Online Material}

www.sciencemag.org/cgi/content/full/1098119/DC1

SOM Text

Figs. S1 to S3

Tables $\mathrm{S} 1$ to $\mathrm{S} 7$

References and Notes

19 March 2004; accepted 27 April 2004

Published online 6 May 2004; 10.1126/science.1098119

Include this information when citing this paper.

Fig. 1. Locations of the 481 ultraconserved elements on the 24 human chromosomes. Each partly exonic element is represent by a thin blue tick mark extending above the chromosome, each non-exonic element by a green tick mark extending below the chromosome, and each possibly exonic element by a black tick mark centered on the chromosome (see text). Purple boxes represent centromeres. By joining two elements into a cluster when they are separated by less than $675 \mathrm{~Kb}$, we obtained 89 local clusters of two or more elements, each of which is boxed and named. Names are taken from a prominent gene or gene-family co-located with the cluster, or by a Drosophila ortholog or mRNA accession if no HUGO named gene was available. Among the cluster representatives there is a distinct enrichment for non-exonic elements and for developmental genes, suggesting that many of these clusters may be part of distal enhancers or "global control loci" analogous to those studied in association with HOXD (38) or DACH (21). One possible such cluster, near the ARX gene, is shown in more detail in the inset at the bottom of the figure (see text). Here known genes are shown 
in blue (tall boxes for coding exons, shorter boxes for UTR, and hatched lines for introns) and ultraconserved elements are shown below them.

Fig. 2. Annotation enrichment in Type I and Type II genes. In the top half of the figure, labeled "Type I genes" (see text), the maroon bars ("observed") give the numbers of type I genes that are annotated in the Gene Ontology (19) with molecular function "RNA binding" or "DNA binding", biological process "RNA splicing" or "regulation of transcription", or are annotated in InterPro (20) as containing the motifs "RNA Recognition Motif" or "Homeobox". The blue bars ("expected") give the number of genes that one would expect to obtain if the same number of genes (111 genes for type I) were chosen at random among all genes annotated in the relevant database. The bottom half of the table gives similar information for type II genes. It is apparent that type I genes are enriched for RNA-related functions, while type II are not. Both types are enriched for DNArelated functions, but the type II genes are more enriched. See text for estimates of the significance of these enrichments. 


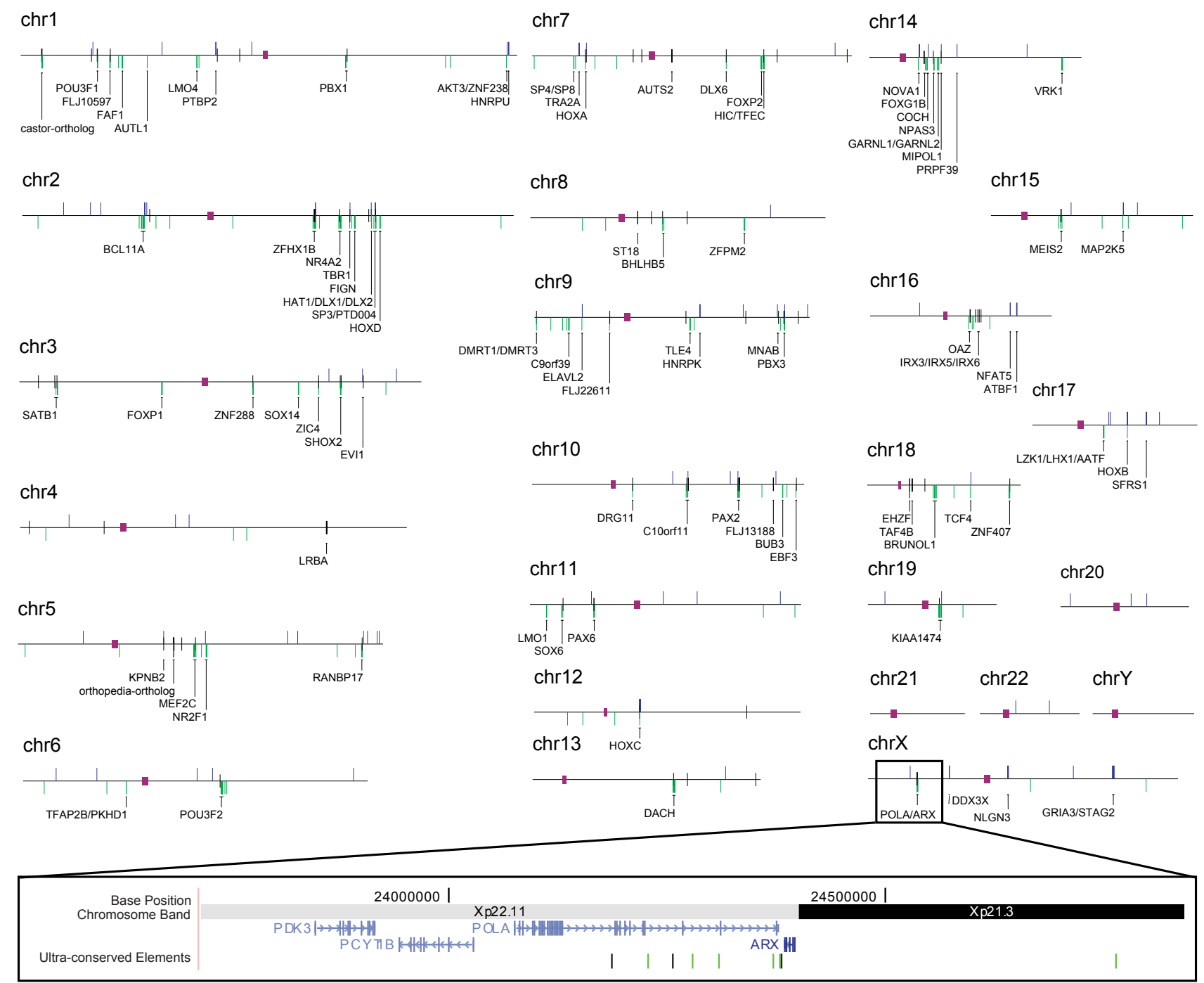




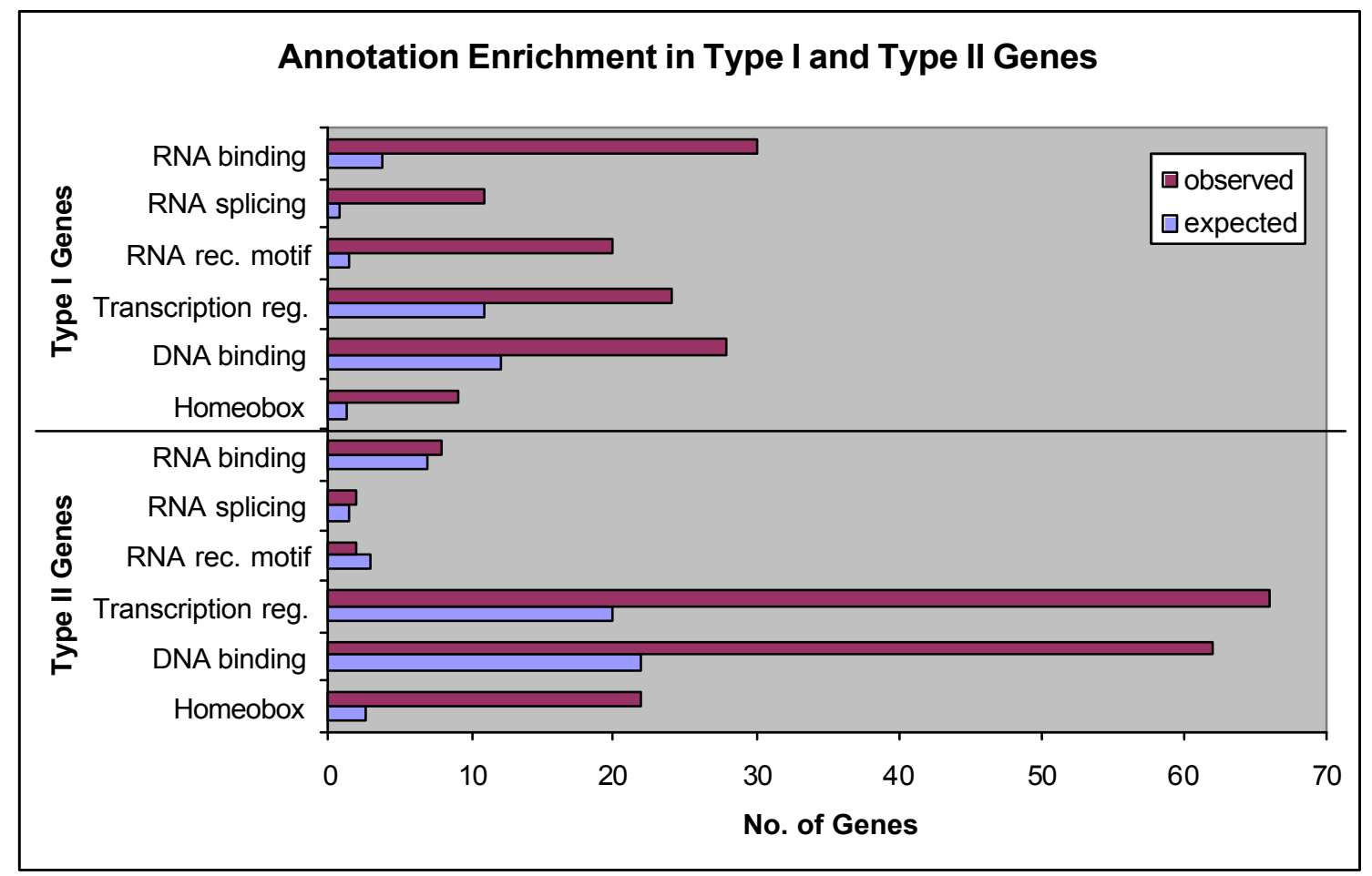

